\title{
Correction to: An Overview of Pair-Potential Functions for Multi-objective Optimization
}

\author{
Jesús Guillermo Falcón-Cardona (iD, Edgar Covantes Osuna (D, \\ and Carlos A. Coello Coello iD
}

\section{Correction to:}

Chapter "An Overview of Pair-Potential Functions

for Multi-objective Optimization" in: H. Ishibuchi et al. (Eds.):

Evolutionary Multi-Criterion Optimization, LNCS 12654,

https://doi.org/10.1007/978-3-030-72062-9_32

The original version of this chapter was revised. Two equations in section 2.1 have been corrected.

$$
\begin{gathered}
\mathcal{K}^{C O U}(\boldsymbol{u}, \boldsymbol{v})=\frac{q_{1} q_{2}}{4 \pi \epsilon_{0}} \cdot \frac{1}{\|\boldsymbol{u}-\boldsymbol{v}\|^{2}} \\
\mathcal{K}^{\mathrm{PT}}(\boldsymbol{u}, \boldsymbol{v})=\frac{V_{1}}{\sin ^{2}(\alpha\|\boldsymbol{u}-\boldsymbol{v}\|)}+\frac{V_{2}}{\cos ^{2}(\alpha\|\boldsymbol{u}-\boldsymbol{v}\|)}
\end{gathered}
$$

